\title{
Beckwith-Wiedemann syndrome: imprinting in clusters revisited
}

\author{
Eamonn R. Maher ${ }^{1}$ and Wolf Reik ${ }^{2}$ \\ ${ }^{1}$ Section of Medical and Molecular Genetics, Department of Paediatrics and Child Health, \\ University of Birmingham, Birmingham B15 2TT, United Kingdom \\ ${ }^{2}$ Laboratory of Developmental Genetics and Imprinting, The Babraham Institute, Cambridge CB2 4AT, United Kingdom \\ Address correspondence to: Eamonn R. Maher, Section of Medical and Molecular Genetics, \\ Department of Paediatrics and Child Health, University of Birmingham, The Medical School, Edgbaston, \\ Birmingham B15 2TT, United Kingdom. Phone: 44-121-627-2630; Fax: 44-121-627-2618; E-mail: ermaher@hgmp.mrc.ac.uk.
}

\section{Introduction}

Genomic imprinting, a process that causes genes to be expressed according to their parental origin, affects a minority of human genes, probably less than 1,000. Nevertheless, this class of epigenetic modification acts on many genes with critical roles in growth and development, and disordered imprinting is implicated in genetic disease and in many cancers. Various theories have been proposed to explain the evolution of imprinting in mammals. The most popular is the Haig parental conflict model, which holds that imprinting evolved as a result of opposing interests of the maternal and paternal genomes. Thus, in polygamous species, paternally derived genes will favor fetal growth at the expense of depleting maternal resources and disadvantaging further offspring. In contrast, the maternal genes will oppose the paternal effect and conserve resources to ensure the fitness of the mother and future offspring. This model predicts that although paternally expressed imprinted genes should promote growth, maternally expressed genes should have opposite effects. Many, but not all, imprinted genes identified to date conform to these predictions (1).

The mechanisms by which imprinting is established and maintained are the subject of intense investigation (2). The process must involve a reversible epigenetic marking, and a number of features of imprinted genes have been described. Thus, differential methylation of maternal and paternal alleles is a major characteristic of imprinted genes, and defects in methylation processes (e.g., methyltransferase deficiency) disrupt normal genomic imprinting. Parental allele-specific alterations in the chromosome environment of imprinted genes are revealed by the presence of asynchronous DNA replication and differences in chromatin structure and modification (e.g., histone acetylation).

A striking feature of imprinted genes in mammals is their tendency to cluster in the genome. The arrangement of coordinately or oppositely imprinted genes within a cluster offers insights into the mechanisms by which cells establish and maintain appropriate imprints on functionally related genes. Here, we discuss the organization and function of a major imprinted gene cluster, occurring on human chromosome 11p15.5, that has been implicated in the imprinting disorder Beckwith-Wiedemann syndrome (BWS) and in a variety of human cancers including Wilms' tumor $(3,4)$.
Because the regulation of imprinted genes in the homologous region in the mouse (distal region of chromosome 7) is broadly conserved between mice and humans, experiments in mice have complemented molecular analyses of BWS pathology and have contributed greatly to our current knowledge of mammalian genomic imprinting mechanisms. In this review, we consider the mechanisms and role of genomic imprinting in human development and in BWS pathogenesis, drawing on research on the human and the mouse clusters of imprinted genes.

\section{Clinical genetics}

BWS is a congenital overgrowth syndrome characterized by pre- and postnatal overgrowth, macroglossia, and anterior abdominal wall defects. Additional, but variable, complications include organomegaly, hypoglycemia, hemihypertrophy, genitourinary abnormalities, and, in about $5 \%$ of children, embryonal tumors (most frequently Wilms' tumor) (3). The genetics of BWS are complex, but evidence for genomic imprinting effects is present for each of the 3 major subgroups of patients (familial, sporadic, and those with chromosome anomalies) (see ref. 3 and references cited therein). Familial clustering accounts for approximately $15 \%$ of BWS cases. The great majority of sporadic and familial cases show no cytogenetic abnormality, but approximately $2 \%$ carry duplications, inversions, or translocations affecting distal 11 p. Because BWS pathogenesis depends, in each of these subgroups of patients, on parent-of-origin effects, it appears that imprinting of 1 or more genes in this region is disrupted. Thus, in familial cases, the risk to offspring depends on the sex of the transmitting parent, with maternal transmission associated with greatly increased penetrance. Approximately 20\% of sporadic cases demonstrate paternal uniparental disomy (UPD) for chromosome 11p15.5, and many other sporadic cases show altered imprinting of BWS candidate genes (see below). To date, all cases of UPD in BWS have resulted from postzygotic mitotic recombination and are mosaic for paternal isodisomy. Among the patients with BWS who have cytogenetic abnormalities - chromosome 11p15.5 duplications, inversions in distal $11 \mathrm{p}$, or balanced translocations that break in this region - genomic imprinting effects are evident in the parental origin of the affected chro- 


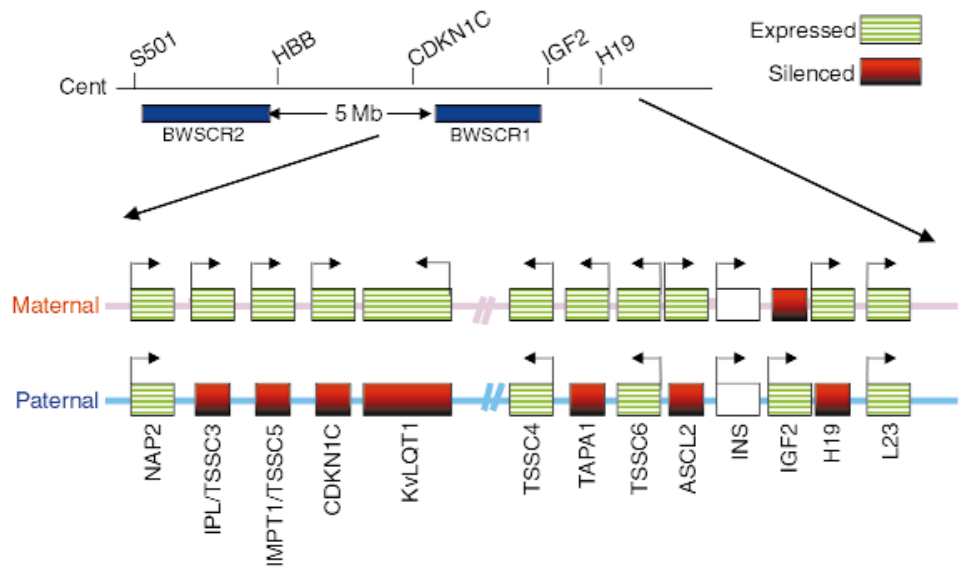

Figure 1

The imprinting cluster on human chromosome 11p15.5. The distal breakpoint cluster region (BWSCR1) is indicated. Breakpoints within BWSCR1 disrupt KvLQT1. Solid red symbols indicate allelic silencing; green/white symbols indicate allelic transcription. The imprinting status of the INS (insulin) gene has not been defined in humans. Further details are included in the text. mosomes. Duplications in this group of patients are invariably derived from the patient's father, whereas inversions and translocations always involve the maternal homologue.

Maternally inherited rearrangements associated with BWS have been mapped to 3 distinct breakpoint cluster regions (BWSCRs). The most frequent of these is BWSCR1, and breakpoints in this region interrupt the KvLQT1 (KCNQ1) gene and map at least $200 \mathrm{~kb}$ proximal to IGF2 (Figure 1) (5). In addition, 2 less-frequent breakpoint cluster regions, BWSCR2 and BWSCR3, map approximately $5 \mathrm{Mb}$ and $7 \mathrm{Mb}$ centromeric to BWSCR1 (6).

\section{Chromosome 11p15.5 and BWS candidate genes}

A variety of strategies have been used to isolate genes from the imprinted gene cluster between $h N A P 2$ and $L 23$ on chromosome 11p15.5 (Figure 1). Initially, all genes identified from this region were imprinted, but at least 2 apparent exceptions, the nonimprinted TSSC4 and TSSC6 genes, are now known (7). These genes appear to subdivide the imprinted cluster region into 2 major subgroups, with proximal (containing CDKN1C, KvLQT1, IPL/TSSC3, and IMPT1/TSSC5 genes) and distal (containing IGF2, INS, and H19 genes) clusters separated by nonimprinted genes. This observation may oversimplify matters, as some genes are subject to partial, tissuespecific, or developmental stage-dependent imprinting.

Several chromosome $11 \mathrm{p} 15.5$ genes may be implicated in familial and sporadic forms of BWS. The most intensively studied of these candidates are CDKN1C, H19, IGF2, and KVLQT1 (KCNQ1). CDKN1C encodes a cyclin-dependent kinase inhibitor belonging to the CIP family of cell-cycle regulators. Overexpression of CDKN1C, also known as p57 KIP2, can arrest cells in G1. Cells that have exited the cell cycle express this protein, and 2 germline CDKN1C mutations found in patients with BWS cause loss of cell-cycle inhibition (8). H19 encodes an untranslated RNA of unknown function (but see the Perspective by Tycko in this series for a discussion of H19 in tumorigenesis). IGF2 encodes a fetal growth factor, and overgrowth in BWS is restricted to tissues in which IGF2 is expressed. KVLQT1 (KCNQ1) encodes a potassium channel that is mutated in long QT syndrome (a dominantly inherited cardiac conduc- tion defect) and in Jervell-Lange-Nielson syndrome (an autosomal recessive disorder characterized by deafness and cardiac conduction defects) (9). Although KVLQT1 is disrupted by BWSCR1 breakpoints, the KVLQT1 gene product does not appear to have a role in BWS. However, KVLQT1 encodes multiple transcripts, and a role for an untranslated transcript in imprinting cannot be excluded.

The IGF2 and H19 genes are closely linked and oppositely imprinted. The relationship between IGF2 and H19 imprinting has been investigated intensively in mouse experiments (see ref. 10 and references cited therein). The most common explanation for reciprocal imprinting of IGF2 and $H 19$ postulates enhancer competition between these genes. According to this model, a shared enhancer downstream of H19 drives H19 RNA expression from the maternal allele. On the paternal allele, however, methylation of the H19 promoter allows the enhancer to interact instead with the adjacent IGF2 gene. Recently, an alternative model has been suggested in which an epigenetic mark upstream of $H 19$ acts as a boundary element on the unmethylated maternal chromosome (11). The imprinting of other genes within the cluster, such as Cdkn1c and Kvlqt1, appears to be independent of $H 19$ imprinting (12).

\section{Molecular pathology of BWS}

The observation that paternal UPD causes BWS is consistent with the existence, on 11p15.5, of either paternally expressed genes that act as growth promoters or maternally expressed genes that suppress growth. As discussed later here, genes of both descriptions may be found within this chromosomal region, and there is strong evidence for both of these etiologic models. Because the entire BWS candidate region has been included in the disomic segment of all known UPD-associated cases, it has not been possible to reduce the range of candidate genes by analyzing these individuals. However, a subset of sporadic and familial BWS cases carry germline mutations in $C D K N 1 C$, providing unequivocal evidence for the involvement of a single gene in at least some affected people (13). Among familial cases, approximately $40 \%$ carry mutations in $C D K N 1 C$, but the incidence of such mutations among sporadic cases is much lower, at approximately $5 \%(14)$. CDKN1C is maternally 
expressed, with a low level of expression from the paternal allele in humans but not in mice. Thus, the parentof-origin effects on penetrance described in familial BWS are consistent with predominantly maternal allele expression of CDKN1C.

Although mutations in CDKN1C may be sufficient to cause disease in some individuals, the absence of such mutations in most sporadic cases of BWS indicates that other pathogenic mechanisms must exist. The paternally expressed fetal growth-promoting gene IGF2 has long been considered a strong candidate for BWS. Two groups have reported that abnormal, biallelic expression of IGF2 (often designated as loss of imprinting, or LOI) occurs in the majority of sporadic BWS cases $(15,16)$. However, in a further series, the frequency of IGF2 LOI was reported to be less than $10 \%$, differences that may, in part, reflect variable patient selection criteria (17).

Epigenetic analysis of patients with BWS with LOI of IGF2 indicates that at least 2 imprinting centers (BWSIC1 and BWSIC2) exist on 11p15.5 (17-19; see also Table 1). Mouse experimental data linking Igf2 and H19 imprinting had already implicated defects at an "imprinting center" in the misexpression of these imprinted genes (10). Likewise, a small subgroup of sporadic BWS cases shows biallelic expression of IGF2 and silencing of H19, accompanied by a paternal methylation pattern on both chromosomes, suggesting that an imprinting control region (BWSIC1) is inactive in such individuals (18). Candidate regulatory regions proposed from experiments with mice include a CpGrich site immediately upstream of $\mathrm{H} 19$ and an intergenic region between $I g f 2$ and $H 19$ (11). The analogous region immediately upstream of the human $H 19$ gene, which is thought to be the site of the primary (gametic) H19 imprint, is in fact biallelically methylated in this subgroup of patients with BWS. So this pathway for "LOI" of IGF2 may be more accurately described, in a mechanistic sense, as a "gain of imprinting" at H19.

In another pair of imprinting disorders, familial Prader-Willi syndrome (PWS) and Angelman syndrome (AS) deletions of upstream untranslated exons of the SNRPN gene have helped to define imprinting control regions (20), but, to date, no germline deletions or mutations have been identified in patients with BWS who have BWSIC1 defects. However, some sporadic
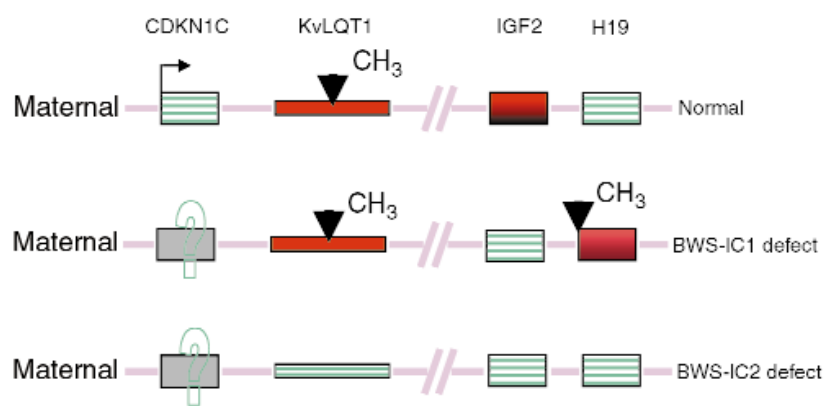

Figure 2

Maternal epigenotype in normal controls and in patients with BWS who have BWSIC1 and BWSIC2 defects. Solid red symbols indicate allelic silencing; green/white symbols indicate allelic transcription. $\mathrm{CH}_{3}$ indicates methylation.
PWS and AS cases with imprinting center defects do not have deletions and probably represent epigenetic errors (21). All BWSIC1 cases described to date are sporadic, and they may also represent similar "epimutations" (our unpublished observations).

Brown et al. observed LOI for IGF2 in the presence of a normal $H 19$ epigenotype in a patient with BWS who had inherited a chromosome 11p15.5 inversion breakpoint in BWSCR1 (22). These data provided the first evidence of an additional, H19-independent mechanism for IGF2 imprinting in humans and suggested that another imprinting center (BWSIC2) must exist at or centromeric to the breakpoint. Most sporadic patients with BWS show IGF2 LOI and a normal H19 epigenotype, suggesting a possible BWSIC2 defect. Consistent with this possibility, Smilinich et al. (19) recently reported that patients with BWS who have H19 hypermethylation - and, hence, BWSIC1 defects - exhibited normal methylation patterns in a differentially methylated region (DMR) near the KVLQT gene (KvDMR1; see below). In contrast, patients with normal H19 methylation patterns frequently showed loss of methylation on the maternal allele at KvDMR1 (19) (Figure 2). In 2 of 2 informative patients, KvDMR1 demethylation was associated with IGF2 LOI and normal H19 epigenotype. Loss of maternal allele KvDMR1 methylation correlated with expression of an antisense transcript, KCNQ1OT, from the maternal copy of this gene.

These findings are consistent with 2 imprinting control centers in 11p15.5: (a) a distal BWSIC1 regulating IGF2 and $H 19$ imprinting and (b) a more centromeric BWSIC2 regulating the imprinting of IGF2 and the expression of this antisense transcript. Lee et al. (17) reported similar results, but found that KvDMR1 demethylation was not associated with IGF2 LOI in all cases, although it was consistently associated with biallelic expression of the KCNQ1OT transcript (which they term LIT1). Thus, BWSIC2 regulation of IGF2 may be polymorphic, or LOI of IGF2 may be sensitive to the exact nature of the BWSIC2 defect. One important issue that has not yet been resolved is the effect of BWSIC2 defects on maternal CDKN1C expression (Table 1). In the mouse, $\mathrm{C} d k n 1 c$ expression requires a maternal germline imprint (23), although maternal germline methylation has not yet been found in or near the gene. Recent transgenic experiments have failed to identify a functional imprinting signal within $35 \mathrm{~kb}$ surrounding the CDKN1C gene (24). It would therefore not be surprising if CDKN1C imprinting were influenced by the BWSIC2, and a preliminary genotype-phenotype correlation is consistent with this hypothesis, as discussed later here.

\section{Mouse models of BWS and genotype-phenotype correlations}

Several genetically engineered mouse strains have been developed that recapitulate aspects of the BWS phenotype and of BWS candidate gene regulation. Thus, overexpression of IGF2 in transgenic mice causes overgrowth, macroglossia, and organomegaly in a dose-dependent fashion $(25,26)$. Murine $H 19$ deletions, likewise, mimic the epigenetic effects of 
Table 1

Molecular subgroups of BWS: observed (in bold) and predicted (in italics) characteristics for IGF2, H19, and CDKN1C. (Data are derived from references $2,13,15,17,18$, and 21 .)

BWS subgroup

\begin{tabular}{|c|c|c|c|c|c|c|}
\hline & UPD & $\begin{array}{c}\text { Maternal } \\
\text { rearrangement }\end{array}$ & $\begin{array}{l}\text { Paternal } \\
\text { duplication }\end{array}$ & $\begin{array}{l}\text { CDKN1C } \\
\text { mutation }\end{array}$ & $\begin{array}{c}\text { BWSIC1 } \\
\text { defect }\end{array}$ & $\begin{array}{c}\text { BWSIC2 } \\
\text { defect }\end{array}$ \\
\hline $\begin{array}{l}\text { IGF2 } \\
\text { expression }\end{array}$ & Increased & Biallelic & Increased & Unaltered & Biallelic & $\begin{array}{c}\text { Biallelic } \\
\text { (variable) }\end{array}$ \\
\hline $\begin{array}{l}\text { IGF2 } \\
\text { methylation }\end{array}$ & Increased & Normal & Increased & Normal & Increased & Normal \\
\hline $\begin{array}{l}\text { H19 } \\
\text { expression }\end{array}$ & Reduced & Unaltered & Unaltered & Unaltered & Reduced & Unaltered \\
\hline $\begin{array}{l}\text { H19 } \\
\text { methylation }\end{array}$ & Increased & Normal & Increased & Normal & Increased & Normal \\
\hline $\begin{array}{l}\text { KvDMR1 } \\
\text { methylation }\end{array}$ & Reduced & & & Normal & Normal & Normal \\
\hline CDKN1C & Reduced expression & & Unaltered & Mutated & Unaltered & Reduced? \\
\hline $\begin{array}{l}\text { Replication } \\
\text { timing }\end{array}$ & Altered & Altered & & & Normal & \\
\hline Frequency & $20 \%$ sporadic & $2 \%$ & $2 \%$ & $\begin{array}{l}40 \% \text { familial } \\
5 \% \text { sporadic }\end{array}$ & $5-10 \%$ sporadic & $\sim 40 \%$ sporadic \\
\hline
\end{tabular}

BWSIC1 defects $(11,18)$. Although the earliest definitive evidence for a second imprinting center (BWSIC2) arose from studies of patients with BWS, the observation that $H 19$ deletions do not affect imprinting of Cdkn1c and Kvlqt1 in the mouse (12) provided independent data compatible with the existence of such a regulatory site. Third, a Cdkn1c knockout mouse, a model for BWS cases with germline CDKN1C mutations (27), displays anterior abdominal wall defects, renal medullary dysplasia, and adrenal cortical cytomegaly, but lacks other features of BWS, notably prenatal overgrowth. It remains to be established whether mouse and human fetuses respond fundamentally differently to lack of CDKN1C, orperhaps more likely-whether the BWS mutations are not null alleles but the mouse knockout is. In any event, these findings suggest a link between exomphalos and CDKN1C inactivation in BWS. However, Eggenschwiler et al. (26), who developed yet another mouse model of BWS by crossing mice with $\mathrm{H} 19$ and $\operatorname{Igf} 2 r$ mutations, identified another mechanism that may account for this phenotype. As a result of Igf2 LOI (due to the H19 deletion) and reduced clearance of circulating IGF2 protein, the resulting double knockout exhibited greater increases in levels of IGF2 than did the Igf2 transgenic mice described here (25), and they displayed both overgrowth and exomphalos.

The variable molecular pathology of BWS provides scope for genotype-phenotype correlations. Analysis of uniparental disomy cases demonstrated a close association with hemihypertrophy (presumably resulting from differing degrees of mosaicism between the 2 sides). We have also defined a high incidence of exomphalos (9/11, $81 \%)$ in patients with BWS with germline CDKN1C mutations (14). Exomphalos is not a feature of patients with BWS who have UPD or BWSIC1 defects, and the absence of exomphalos in UPD cases probably reflects a milder phenotype because of mosaicism.
Phenotypic differences between CDKN1C mutation cases and patients with BWSIC1 defect strongly suggest that, although IGF2 overexpression and CDKN1C inactivation may perturb the same signaling pathway, their effects are not equivalent, perhaps because IGF2 and CDKN1C antagonize each other's effects, but only in certain tissues or at certain developmental stages. The genotype-phenotype correlations in BWS are broadly consistent with the differing phenotypes of the IGF2 overexpression and CDKN1C knockout mouse models of BWS. In addition, we note that children with BWS with chromosome 11 duplications (predicted to have increased IGF2 expression and normal CDKN1C expression) do not have exomphalos. Historically, anterior wall defects in BWS have been attributed to the mechanical effects of intra-abdominal organomegaly. However the close association between exomphalos and CDKN1C inactivation suggests that the developmental defect reflects alterations in cell growth, differentiation, or death in the abdominal wall. Preliminary genotype-phenotype analysis of BWSIC2 defect cases suggest that exomphalos is relatively common (E.R. Maher, unpublished observations). According to our model of the pathogenesis of exomphalos in BWS, this observation would predict altered CDKN1C expression in BWSIC2 cases.

$\mathrm{BWS}$ is genetically heterogeneous, and it appears that phenotypic differences between molecular subgroups reflect differential alterations in BWS candidate genes. To date, there is strong evidence for both IGF2 and CDKN1C in the pathogenesis of BWS and these genes may be linked to particular features of BWS (e.g., exomphalos and CDKN1C inactivation; overgrowth and IGF2 overexpression in mice and, possibly, in humans). The significance of H19 in determining the BWS phenotype is unclear. A tentative association between reduced H19 expression in BWS and Wilms' tumor susceptibility has been suggested (4). Our own data would be consistent 
with this hypothesis, as BWS cases with Wilms' tumor have had UPD or BWSIC1 defects. However the numbers are too small to allow meaningful statistical comparison between subgroups, so this aspect requires further investigation.

The phenotype of the X-linked overgrowth disorder, Simpson-Golabi-Behmel syndrome (SGBS) overlaps with BWS. Many cases of SGBS result from defects in the glypican-3 (GPC3) gene (28). Although glypican-3 has been suggested to negatively regulate IGF2, direct evidence for this hypothesis is not available, and GPC3 mutations may disrupt other signaling pathways (29). Nevertheless, the phenotypic similarities between BWS and SGBS suggest functional relationships between IGF2, CDKN1C, and GPC3 gene products.

\section{Molecular mechanisms of normal and abnormal imprint regulation}

The mechanisms of introduction of imprints in germ cells, their maintenance in the embryo, and the reading of the imprints and their conversion into differential gene activity are still largely unknown $(2,10)$. However, a few elements have been defined that are important for the control of imprinting and that may account for the aberrant gene regulation in BWS. Apart from enhancers and promoters, 2 distinct, but possibly related, elements have been described that may contribute to allele-specific gene expression: antisense promoters and boundary elements. These elements are epigenetically regulated by DNA methylation or chromatin modifications.

Parental allele-specific methylation differences are a frequent, but not invariable, feature of imprinted genes; 11 p15.5 includes several DMRs, including the paternally methylated sites in the IGF2 gene (equivalent to mouse DMR2), the DMR upstream of H19, and the maternally methylated KvDMR1 site within the KvLQT1 gene (17-19) (Figure 2). Untranslated antisense transcripts, a recently recognized characteristic of imprinted regions, are also found for multiple genes in the BWS region. An antisense transcript (air) from the murine Igf2r gene is expressed from the paternal allele, which bears a methylated promoter and is silent with respect to sense transcript expression. However, on the maternal allele, methylation of an intragenic site is associated with repression of the antisense transcript and expression of the sense Igf $2 \mathrm{r}$ mRNA (30). Thus, although the relationship between sense and antisense expression remains obscure, antisense Igf $2 \mathrm{r}$ RNA is linked with Iff $r$ imprinting in a process that has been termed expression competition (31). In addition, a KvLQT1 antisense transcript (KCNQ1OT or LIT1) has been implicated in BWS $(17,19)$. How antisense transcription prevents sense transcripts is not clear.

A chromatin boundary element in the imprinting control region upstream of $H 19$ apparently regulates access of enhancers to nearby promoters $(11,32)$. Here, the demethylated boundary region restricts access of the $H 19$ endoderm enhancers, which are located $3^{\prime}$ of $H 19$, to its promoter. When methylated, the boundary is "open" and allows the enhancers access to Igf 2 . Although promoter methylation affects local gene expression, boundaries that open and close appear to contribute to broader regional controls.

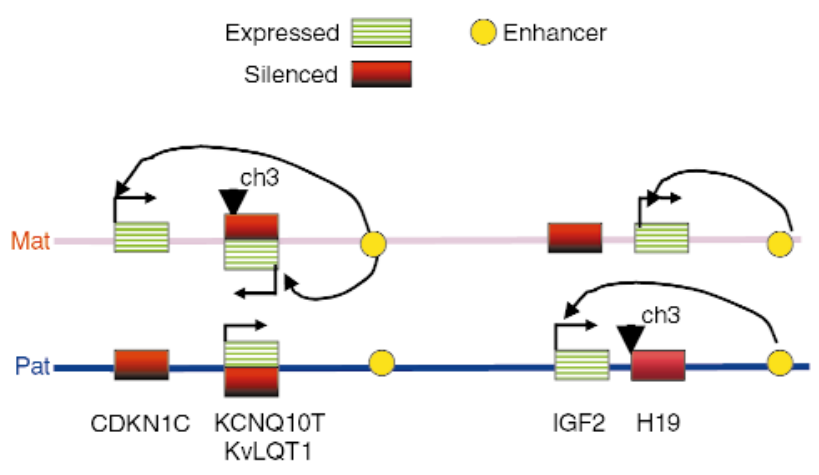

Figure 3

A speculative model for imprinting of genes in the BWS cluster on chromosome 11p15.5. Shared enhancers exist for IGF2 and H19 and are proposed for CDKN1C and KvLQT1. Methylation of the H19 DMR silences $H 19$ and activates IGF2. Methylation of KvDMR1 is proposed to silence KCNQ1OT and to activate CDKN1C and KVLQT1.

A very speculative model for gene regulation within the BWS cluster can be envisaged, using these building blocks (Figure 3). The KvDMR1 regulates antisense expression and is a boundary, which is open when methylated (as in the normal maternal chromosome). The proposed enhancers for both CDKN1C and $K v L Q T 1$ are located $3^{\prime}$ of this boundary. On the maternal allele, these enhancers are active and both CDKN1C and KvLQT1 are expressed, consistent with the requirement for a maternal germline imprint for CDKN1C activity. The antisense transcript (KCNQ1OT) is made on the paternal allele and blocks paternal expression of KvLQT1. At the other end of the cluster, methylation of the H19 DMR on the paternal allele permits transcription of Igf2 by an enhancer residing $3^{\prime}$ of both genes. Methylation of $\mathrm{H} 19$ on the maternal chromosome (e.g., in BWSIC1 defect cases) then leads to LOI of IGF2. In BWSIC2 defects, demethylation of KvDMR1 on the maternal chromosome (or BWSCR1 translocations) would be predicted, under this model, to lead to silencing of CDKN1C. How this could also lead to LOI of IGF2 is not clear.

Imprinting defects in 2 principal locations (e.g., BWSIC1, BWSIC2) can explain the molecular pathology in a proportion of patients. However, it is likely that defects in other elements will be found in other classes of patients. In addition, levels of epigenetic information other than methylation can also be affected. It is now clear that one of the ways methylation can exert its repressive effects is through deacetylation of histones, which results in chromatin compaction that is less efficiently transcribed (33). Hence, defects in local or regional patterns of histone acetylation could also lead to altered expression of imprinted genes. This mechanism needs to be investigated in BWS.

\section{Conclusions}

Although the IGF2 and H19 genes are well characterized, the downstream effectors of IGF2 have not been elucidated in detail, and the function of H19 RNA is enigmatic. It is reasonable to speculate that the link 
between the functions of the IGF2 and CDKN1C gene products that are revealed by their involvement in BWS might turn out to be the first of many examples of functional interactions between genes within an imprinted gene cluster. Thus, clustering of imprinted genes enables coordinate regulation of imprinting across large domains and more local mechanisms within specific regions of the domain. Thus, on $11 \mathrm{p} 15.5$, BWSIC1 may provide a local mechanism for regulating IGF2 and H19 imprinting, whereas a second mechanism, possibly related to chromatin structure, regulates imprinting over a larger distance.

\section{Acknowledgments}

We apologize to those authors whose work we were unable to cite because of space restrictions. We thank the many colleagues who referred patients, and P. Schofield for his contribution to BWS research. We are grateful to the Wellcome Trust (E.R. Maher), Action Research (W. Reik, E.R. Maher), Cancer Research Campaign (W. Reik), BBSRC (W. Riek), and The Birth Defects Foundation (E.R. Maher) for financial support.

1. Hurst, L.D., and McVean, G.T. 1997. Growth effects of uniparental disomies and the conflict theory of genomic imprinting. Trends Genet. 11:436-443.

2. Constancia, M., Pickard, B., Kelsey, G., and Reik, W. 1998. Imprinting mechanisms. Genome Res. 8:881-900.

3. Reik, W., and Maher, E.R. 1997. Imprinting in clusters: lessons from Beckwith-Wiedemann syndrome. Trends Genet. 3:330-334.

4. Tycko, B. 2000. Genomic imprinting and human neoplasia. In DNA alterations in cancer: genetic and epigenetic changes. M. Ehrlich, editor. Eaton Publishing. Natick, UK.

5. Lee, M.P., Hu, R.-J., Johnson, L.A., and Feinberg, A.P. 1997. Human KVLQT1 gene shows tissue specific imprinting and encompasses Beckwith-Weidemann syndrome chromosomal rearrangements. Nat. Genet. 15:181-185.

6. Redeker, E., et al. 1995. Physical mapping of 3 candidate tumor suppressor genes relative to BWS associated chromosomal break points at 11p15.3. Cytogenet. Cell Genet. 68:222-225.

7. Lee, M.P., et al. 1999. Two novel genes in the center of the $11 \mathrm{p} 15$ imprinted domain escape genomic imprinting. Hum. Mol. Genet. 8:683-690.

8. Bhuiyan, Z.A., et al.1999. Functional analysis of the p57KIP2 gene mutation in Beckwith-Wiedemann syndrome. Hum. Genet. 104:205-210.

9. Neyroud, N., et al. 1997. A novel mutation in the potassium channel gene KVLQT1 causes the Jervell and Lange-Nielsen cardioauditory syndrome. Nat. Genet. 15:186-189.

10. Bartolomei, M.S., and Tilghman, S.M. 1997. Genomic imprinting in mammals. Annu. Rev. Genet. 31:493-525.

11. Schmidt, J.V., Levorse, J.M., and Tilghman, S.M. 1999. Enhancer competition between $\mathrm{H} 19$ and Igf2 does not mediate their imprinting. Proc. Natl. Acad. Sci. USA. 96:9733-9738.

12. Caspary, T., Cleary, M.A., Baker, C.C., Guan, X.-J., and Tilghman, S.M.
1998. Multiple mechanisms regulate imprinting of the mouse distal chromosome 7 gene cluster. Mol. Cell. Biol. 18:3466-3474.

13. Hatada, I., et al. 1996. An imprinted gene p57Kip2 is mutated in Beckwith-Wiedemann syndrome. Nat. Genet. 14:171-174.

14. Lam, W.W.K., et al. 1999. Analysis of germline CDKN1C (p57KIP2) mutations in familial and sporadic Beckwith-Wiedemann syndrome (BWS) provides a novel genotype-phenotype correlation. J. Med. Genet. 36:518-523.

15. Weksberg, R., Shen, D.R., Fei, Y.L., Song, Q.L., and Squire, J. 1993. Disruption of insulin-like growth factor 2 imprinting in Beckwith-Wiedemann syndrome. Nat. Genet. 5:143-150.

16. Joyce, J.A., et al. 1997. Imprinting of IGF2 and H19: lack of reciprocity in sporadic Beckwith-Wiedemann syndrome. Hum. Mol. Genet. 6:1543-1548.

17. Lee, M.P., et al. 1999. Loss of imprinting of a paternally expressed transcript, with antisense orientation to KVLQT1, occurs frequently in Beckwith-Wiedemann syndrome and is independent of insulin-like growth factor II imprinting. Proc. Natl. Acad. Sci. USA. 96:5203-5208.

18. Reik, W., et al. 1995. Imprinting mutations in the Beckwith-Wiedemann syndrome suggested by an altered imprinting pattern in the IGF2-H19 domain. Hum. Mol. Genet. 4:2379-2385.

19. Smilinich, N.J., et al. 1999. A maternally methylated CpG island in KvLQT1 is associated with an antisense paternal transcript and loss of imprinting in Beckwith-Wiedemann syndrome. Proc. Natl. Acad. Sci. USA. 96:8064-8069.

20. Dittrich, B., et al. 1996. Imprint switching on human chromosome 15 may involve alternative transcripts of the SNRPN gene. Nat. Genet. 14:163-170.

21. Buiting, K., et al. 1998. Sporadic imprinting defects in Prader-Willi syndrome and Angelman syndrome: implications for imprint-switch models, genetic counseling, and prenatal diagnosis. Am. J. Hum. Genet. 63:170-180.

22. Brown, K.W., et al. 1996. Imprinting mutation in the Beckwith-Wiedemann syndrome leads to biallelic IGF2 expression through an $\mathrm{H} 19$ independent pathway. Hum. Mol. Genet. 6:2027-2032.

23. Obata, Y., et al. 1998. Disruption of primary imprinting during oocyte growth leads to the modified expression of imprinted genes during embryogenesis. Development. 125:1553-1560.

24. John, R.M., Hodges, M., Little, P., Barton, S.C., and Surani, M.A. 1999. A human $\mathrm{p} 57(\mathrm{KIP} 2)$ transgene is not activated by passage through the maternal mouse germline. Hum. Mol. Genet. 8:2211-2219.

25. Sun, F.L., Dean, W.L., Kelsey, G., Allen, N.D., and Reik, W. 1997. Transactivation of Igf2 in a mouse model of Beckwith-Wiedemann syndrome. Nature. 389:809-815.

26. Eggenschwiler, J., et al. 1997. Mouse mutant embryos overexpressing IGF-II exhibit phenotypic features of the Beckwith-Wiedemann and Simpson-Golabi-Behmel syndromes. Genes Dev. 11:3128-3142.

27. Zhang, P., et al. 1997. Altered cell differentiation and proliferation in mice lacking p57KIP2 indicates a role in Beckwith-Wiedemann syndrome. Nature. 387:151-158.

28. Pilia, G., et al. 1996. Mutations in GPC3, a glypican gene, cause the Simpson-Golabi-Behmel overgrowth syndrome. Nat. Genet. 12:241-247.

29. Selleck, S.B. 1999. Overgrowth syndromes and the regulation of signaling complexes by proteoglycans. Am. J. Hum. Genet. 64:372-377.

30. Wutz, A., et al. 1997. Imprinted expression of the Igf2r gene depends on an intronic CpG island. Nature. 389:745-749.

31. Barlow, D.P. 1997. Competition: a common motif for the imprinting mechanism? EMBO J. 16:6899-6905.

32. Webber, A.L., Ingram, R.S., Levorse, J.M., and Tilghman, S.M. 1998. Location of enhancers is essential for the imprinting of $\mathrm{H} 19$ and Igf2 genes. Nature. 391:711-715.

33. Ng, H.H., and Bird, A. 1999. DNA methylation and chromatin modification. Curr. Opin. Genet. Dev. 9:158-163. 